\title{
Oral medicinal cannabinoids to relieve symptom burden in the palliative care of patients with advanced cancer: a double- blind, placebo-controlled, randomised clinical trial of efficacy and safety of 1:1 delta-9-tetrahydrocannabinol (THC) and cannabidiol (CBD)
}

Janet Hardy ${ }^{1}$, Alison Haywood ${ }^{2,3}$, Gauri Gogna ${ }^{4}$, Jennifer Martin ${ }^{5,6}$, Patsy Yates ${ }^{7,8}$, Ristan Greer ${ }^{3}$ and Phillip Good ${ }^{9 *}$ (D)

\begin{abstract}
Background: Despite improvements in medical care, patients with advanced cancer still experience substantial symptom distress. There is increasing interest in the use of medicinal cannabinoids but little high-quality evidence to guide clinicians. This study aims to define the role of a 1:1 delta-9-tetrahydrocannabinol/cannabidiol (THC/CBD) cannabinoid preparation in the management of symptom burden in patients with advanced cancer undergoing standard palliative care.

Methods and design: One hundred fifty participants will be recruited from five sites within the Queensland Palliative Care Research Group (QPCRG) and randomly assigned to an active treatment or placebo group. This study is a pragmatic multicentre, randomised, placebo-controlled, two-arm trial of escalating doses of an oral 1:1 THC/ CBD cannabinoid preparation. It will compare efficacy and safety outcomes of a titrated dose $(10 \mathrm{mg} / 10 \mathrm{mg} / \mathrm{mL}$ oral solution formulation, dose range $2.5 \mathrm{mg} / 2.5 \mathrm{mg}-30 \mathrm{mg} / 30 \mathrm{mg} /$ day) against placebo. There is a 2-week patientdetermined titration phase, using escalating doses of 1:1 THC/CBD or placebo, to reach a dose that achieves symptom relief with tolerable side effects. This is then followed by a further 2-week assessment period on the stable dose determined in collaboration with clinicians. The primary objective is to assess the effect of escalating doses of a 1:1 THC/CBD cannabinoid preparation against placebo on change in total symptom score, with secondary objectives including establishing a patient-determined effective dose, the change in total physical and emotional sores, global impression of change, anxiety and depression, opioid use, quality of life and adverse effects. (Continued on next page)
\end{abstract}

\footnotetext{
* Correspondence: Phillip.Good@mater.org.au

${ }^{9}$ Mater Health Services, Mater Research Institute-University of Queensland, St Vincent's Private Hospital, Brisbane, Australia

Full list of author information is available at the end of the article
}

C C The Author(s). 2020 Open Access This article is licensed under a Creative Commons Attribution 4.0 International License, which permits use, sharing, adaptation, distribution and reproduction in any medium or format, as long as you give appropriate credit to the original author(s) and the source, provide a link to the Creative Commons licence, and indicate if changes were made. The images or other third party material in this article are included in the article's Creative Commons. licence, unless indicated otherwise in a credit line to the material. If material is not included in the article's Creative Commons licence and your intended use is not permitted by statutory regulation or exceeds the permitted use, you will need to obtain permission directly from the copyright holder. To view a copy of this licence, visit http://creativecommons.org/licenses/by/4.0/ The Creative Commons Public Domain Dedication waiver (http://creativecommons.org/publicdomain/zero/1.0/) applies to the data made available in this article, unless otherwise stated in a credit line to the data. 
(Continued from previous page)

Discussion: This will be the first placebo-controlled clinical trial to rigorously evaluate the efficacy, safety and acceptability of 1:1 THC/CBD for symptom relief in advanced cancer patients. This study will allow the medical community to have some evidence to present to patients wishing to access cannabis for their symptoms caused by advanced malignancy.

Trial registration: ACTRN, ACTRN12619000037101. Registered on 14 January 2019.

Trial Sponsor: Mater Misericordiae Limited (MML) and Mater Medical Research Institute Limited (MMRI)—Raymond Terrace, South Brisbane, Brisbane, QLD, Australia

Keywords: Cannabis, Cannabidiol, Cancer, Symptom control, RCT, Palliative care, THC

\section{Background}

Despite improvements in medical care, patients with advanced cancer still experience substantial symptom distress [1]. Palliative care aims to take a skilled, holistic approach to improve patients' symptoms and quality of life. Whilst there is a range of analgesic medication available for pain management, the control of many other symptoms (e.g. fatigue, anorexia, weight loss) remains a challenge [2].

There has been increasing interest in the use of medicinal cannabinoids over recent years, particularly for the relief of symptoms in palliative care patients [3]. Recent legislative change in several Australian states provided pathways for the use of medicinal cannabinoids for a range of indications including chemotherapy-induced nausea and vomiting (CINV), resistant epilepsy, pain and spasticity in multiple sclerosis, and symptoms associated with terminal illness [4].

Cannabis contains almost 500 bioactive compounds, including over 70 different cannabinoids [5]. The predominant cannabinoids include delta-9-tetrahydrocannabinol (THC) and cannabidiol (CBD). THC is the main psychoactive component of cannabinoids and is thought to act as a partial agonist on the endocannabinoid system. Potential benefits of THC include analgesia, anti-nausea and muscle relaxation, with potential side effects including intoxication, psychosis, anxiety and sedation. The recommended dose range for oral THC varies from 2.5 to $40 \mathrm{mg} /$ day [6]. In contrast, $\mathrm{CBD}$ is not intoxicating and has a range of anxiolytic, antipsychotic, anti-inflammatory, antioxidative, anti-convulsant and neuroprotective effects [7]. $\mathrm{CBD}$ is also considered to mediate many of the adverse psychotropic effects of THC, although this research is still emerging [8]. CBD has been used with a dosing in the range of 40 to $1280 \mathrm{mg} /$ day orally [9].

There is little high-quality evidence of benefit to date for the use of medicinal cannabinoids. The most recent review from the USA National Academies of Sciences, Engineering, and Medicine found substantial evidence for the use of medicinal cannabinoids for treatment of some types of chronic pain, CINV and spasticity in multiple sclerosis in some patients, with moderate evidence for sleep disorders [10]. Cannabinoid products are licenced for a range of conditions in different countries, with little consistency between countries for indication and dosing [11]. Whilst cannabinoids may have clinical indication, their use is not without potential for substantial harms, and further research is needed to define their role in medical practice $[3,11]$.

There are many unknowns when it comes to prescribing medicinal cannabis [11]. This includes the formulation of the drug (ratios of THC or CBD), the dosing of the drug and the best route of delivery. Ongoing concerns remain around the uncertainty over the optimal formulation, toxicity and abuse potential. The 1:1 THC/ $\mathrm{CBD}$ ratio used in formulations may deliver sufficient $\mathrm{CBD}$ to ameliorate the psychotoxic effects of THC but is unlikely to produce significant CBD therapeutic effects.

To date, there has been no formal examination of the effect of different THC/CBD ratios. We will therefore compare efficacy and safety outcomes of a 1:1 THC/ CBD formulation against placebo, with doses of both THC and CBD escalated to doses previously shown to be safe.

\section{Methods}

\section{Aims and objectives}

This study aims to define the role of cannabinoids in the management of symptoms in patients with advanced cancer, who are receiving standard palliative care. The hypothesis is that medicinal cannabinoids will reduce the total symptom burden in these patients compared to placebo.

The primary objective is to assess the effect of escalating doses of a 1:1 THC/CBD cannabinoid preparation against placebo on total symptom score at day 14 as measured by the Edmonton Symptom Assessment Scale (ESAS) (change in score from baseline). Secondary objectives are to establish a patient-determined effective dose of a 1:1 THC/CBD formulation and assess the effect on symptom scores at days 7, 21 and 28. Other secondary objectives include assessing the change in total physical and emotional sores, global impression of change, anxiety and depression, opioid use and quality 
of life at the same time points and to document adverse effects associated with cannabinoid use.

\section{Study design}

This study is a pragmatic multicentre, randomised, placebo-controlled, two-arm parallel trial of escalating doses of oral THC/CBD $10 \mathrm{mg} / 10 \mathrm{mg} / \mathrm{mL}$ oral solution. There is a 2-week patient-determined titration phase, using escalating doses of a 1:1 THC/CBD combination or placebo, to reach a dose that achieves symptom relief with tolerable side effects. This is followed by a further 2-week assessment period on the stable dose determined in collaboration with clinicians.

Eligible patients will be randomly assigned in equal numbers to one of the two study arms. Randomisation schedules will be developed for each site using random number tables, computer generated at an independent centre. Treatment for each patient will be allocated according to a permuted block randomisation schedule held by the central registry. Block randomisation within each centre will ensure even allocation to each arm in each site. The pharmacy will be notified of a participant, and a completed script with the participant's study ID number will be given to the site pharmacy. The pharmacist will randomise the participant according to the schedule and dispense the medication in a labelled bottle. The participant ID, allocation number, date of request, preparation and dispensing will be recorded in a log maintained by the site pharmacist for each randomisation.

All participants, caregivers, investigators and clinical staff will remain blind to study assignment until trial completion. The code will only be broken in cases of a clinical emergency. An investigator not directly involved in the randomisation of patients will keep an un-blinded copy of the randomisation schedule and will be contacted in the event of the need to un-blind. All study drugs and placebo will be in oil solution form and identical in appearance. All oil solutions will be matched for taste, colour and bottle size to preserve the blinding irrespective of the contents; each participant will receive the oil solution in a prepacked bottle labelled with their individual trial participation ID number and consecutively numbered according to the randomisation scale.

\section{Intervention}

Cannabinoid oils, supplied by LG Pharma, will be used in this study. The decision to use oils was based on a number of factors including product availability, ability to access ongoing supplies, ability to produce matched placebo, regulations surrounding the production and transport of cannabinoid products and cost.

Arm 1 of the trial consists of THC/CBD $10 \mathrm{mg} / 10 \mathrm{mg} /$ $\mathrm{mL}$ oral solution (LG Pharma Pty Ltd) with a dose range of $2.5 \mathrm{mg} / 2.5 \mathrm{mg}-30 \mathrm{mg} / 30 \mathrm{mg} /$ day and arm 2 a matching placebo oral oil solution. Participants are asked to take daily doses as per dosing schedule instructions. The study drug is to commence on the day of baseline assessment, continuing for a maximum of 28 days.

The cannabinoid oils and placebo will be dispensed in identical $50-\mathrm{mL}$ bottles containing a sufficient quantity of oil. Bottles are to be stored at room temperature $\left(25^{\circ} \mathrm{C}\right)$ until used and stored away from light. The product will be dispensed from bottles with a wadded childresistant cap. Participants will be given replacement bottles to complete the study as required. Participants will be educated on how to administer daily doses using supplied 1-mL syringes appropriate for the dose. The participant will complete a daily dosing schedule diary at home to record medication taken. Cannabinoid oil that has been allocated to a participant and not used will be returned to the research nurse/research officer and destroyed by the local pharmacy as per pharmacy guidelines.

Dose titration will be confirmed following regular consultation. Each participant will be advised to increase their dose according to a schedule every 2 days until they are satisfied with their symptom improvement or they experience unacceptable side effects. Dose titration downwards will also be allowed, in consultation with research staff. The participant in collaboration with research staff will define the dose level at which they will continue until the primary outcome point (14 days). Participants will then be given the option of remaining on the blinded oil solution for a further 2 weeks ( 28 days total) for continuing assessment of efficacy and adverse events (continue final dose if perceived to be of benefit and tolerated). Assessment at 28 days following 14 days of stable dose is a secondary end point.

\section{Study setting}

Participants will be recruited from five sites within the Queensland Palliative Care Research Group (QPCRG). These five sites have been chosen from the QPCRG as they have an established research team including research support systems within Palliative Care and have previous experience in trial participation in this setting. It is anticipated that this will be predominately an outpatient study, with these sites having the ability to access this participant population from their established clinic service.

All participants will be given standard palliative care according to the local practice of the recruiting centre [12]. They will continue all current medications including opioids, antiemetics, sedatives and specific anti-cancer therapy (including chemotherapy, immunotherapy and radiotherapy).

\section{Study participants}

Patients who meet the inclusion criteria will have an advanced histologically proven cancer diagnosis (metastatic 
or locally advanced), be known to and be receiving palliative care at the recruiting centre and have an ESAS Total Symptom Distress Score (TSDS) of $\geq 10 / 90$ for cancer-related symptoms and at least one individual ESAS score $\geq 3 / 10$ [13]. They must have a Performance Status AKPS (Australia-modified Karnofsky Scale score) of $\geq 30 / 100$ [14], be aged $\geq 25$ years, have a negative THC urine test at commencement of trial and be able to tolerate oral medications and must be either Englishspeaking or have an interpreter available.

Female participants must have a negative pregnancy urine test at eligibility (only if of reproductive potential) and agree to avoid pregnancy during the study and 12 weeks following the last dose of the study drug. Males must agree to avoid fathering a child and to not donate sperm during the study and for at least 12 weeks following the last dose of the study drug. Further to this, the Alcohol, Smoking and Substance Involvement Screening Test (ASSIST) will be completed [15]. It is designed to determine whether harmful substances are being used and go undetected or become worse. The assessment is comprised of 8 questions assessing, tobacco, alcohol, cocaine, amphetamine-type stimulants, sedatives, hallucinogens, opiates and 'other drugs'.

Exclusion criteria include a history of hypersensitivity to any cannabinoid, unstable untreated cardiovascular disease, severe hepatic impairment (total bilirubin $\geq 1.5$ times the upper limit of the normal range), aspartate aminotransferase (AST) and alanine aminotransferase $(\mathrm{ALT}) \geq 3.0$ times the upper limit of the normal range (subjects with liver metastasis may have an AST and ALT of $\geq 5.0$ times the upper limit of normal), severe renal impairment (eGFR $\leq 20 \mathrm{~mL} / \mathrm{min} / 1.73 \mathrm{~m}^{2}$ ), a history of psychiatric disorders (severe depression or anxiety, personality disorder, psychosis, schizophrenia, firstdegree relative with schizophrenia and/or suicidal ideation), cognitive impairment (St Louis University Mental Status examination (SLUMS) $\leq 20 / 30$ ) [16] and a known substance use disorder (Alcohol, Smoking and Substance Involvement Screening Test (ASSIST) examination scoring $>27$ ) for any substance.

Potential participants will be excluded if they have a history suggesting that drug diversion may be a risk for them or their family/carers, have participated in a trial of a new clinical entity within the last 28 days or had treatment with a new specific anti-cancer agent (chemotherapy, targeted or hormonal therapy) or radiation within the last 7 days.

\section{Consent process}

The process for obtaining consent for this study will be exchanging information between the study staff and potential participants and any other person the participant wishes to include in the discussion. A participant information sheet (PICF) will be provided in written form and will be used as the basis for the discussion. This will cover the purpose, expected procedures, participant requirements, risks, benefits, burdens and side effects that are expected or possible during the study. Participants are specifically informed that due to the nature of this medication and current laws in Queensland, you will NOT be able to drive or operate heavy machinery whilst taking the medication. Potential participants will be given the opportunity (in time and physical capacity) to consider the study and formulate any questions. All questions will be addressed and answered fully. An actual time point for consent will not be specified as this will be determined by the person's physical condition. The consent form is to be completed by trained study team members in accordance with the requirements of the approving ethics committee. The form is to be signed and dated by the participant.

\section{Assessments}

Participants will receive 2 times/weekly research nurse phone calls in the first 2 weeks and outpatient clinic medical review visits at days 7, 14, 21 and 28, with outcome measures recorded at these points.

Symptom burden will be measured using the Edmonton Symptom Assessment Scale (ESAS). Confirmed documented disease status will be assessed at days 14 and 28, where applicable.

A routine haematology and biochemistry screen including liver function tests will be taken at eligibility/ baseline assessment. Blood for C-reactive protein (CRP) as a basic test of inflammation will be taken at baseline, day 14 and day 28 . All consenting participants will have a urine test to confirm nil recent use of THC-related products as a pre-screen. Female participants of childbearing potential will have a urine test to determine pregnancy. At day 14, a urine sample will be collected and stored (frozen) until the completion and unblinding of the study. Samples will be stored on site and transported to central storage in batches over the course of the study. These samples will be for post-trial analysis for evidence of no THC product use during the trial.

Participants will be contacted at day $56(+4$ weeks post last dose) for the purposes of safety follow-up and recording of post-study cannabinoid use. Date of death will be recorded for all participants up to the census point. This protocol has been written in accordance with the Standard Protocol Items: Recommendations for Interventional Trials (SPIRIT) (Table 1).

\section{Outcomes and assessment tools}

The primary outcome is the change from baseline of total ESAS TSDS at day 14. 
Table 1 SPIRIT figure_-study schedule

\begin{tabular}{|c|c|c|c|c|c|c|c|c|}
\hline & Eligibility & $\begin{array}{l}\text { Baseline" } \\
\text { Day 0 } \\
\text { (-Ohrs) }\end{array}$ & $\begin{array}{c}\text { Day } 7 \\
\text { (+/-1 day) } \\
\text { (+168hrs) }\end{array}$ & $\begin{array}{c}\text { Day } 14 \\
(+/-1 \text { day }) \\
(+312 \text { hrs })\end{array}$ & $\begin{array}{c}\text { Day } 21 \\
\text { (+/-1 day) } \\
\text { (+504hrs) }\end{array}$ & $\begin{array}{c}\text { Day } 28 \\
(+/-1 \text { day }) \\
(+672)\end{array}$ & $\begin{array}{c}2 \times \text { weekly calls } \\
\text { day } 1-14\end{array}$ & $\begin{array}{l}\text { Follow up phone } \\
\text { call day } 56 \text { after } \\
\text { completion of } \\
\text { study drug }\end{array}$ \\
\hline \multicolumn{9}{|l|}{ ENROLLMENT } \\
\hline Eligibility screen & $\mathrm{x}$ & & & & & & & \\
\hline Informed Consent & & $\mathrm{x}$ & & & & & & \\
\hline Allocation & & $x$ & & & & & & \\
\hline Urine cannabinoid screen & $\mathrm{x}$ & & & $\begin{array}{c}\mathrm{x} \\
\text { (to be frozen) }\end{array}$ & & & & \\
\hline Urine pregnancy test & $\mathrm{x}$ & & & & & & & \\
\hline Haematology \& Biochemistry screen (FBC/elFT) & $x$ & & & $x$ & & & & \\
\hline C-reactive protein (CRP) & & $\mathrm{x}$ & & $x$ & & $\mathrm{x}$ & & \\
\hline \multicolumn{9}{|l|}{ Interventions } \\
\hline \multicolumn{9}{|l|}{$\mathrm{THC} / \mathrm{CBD}$} \\
\hline Placebo & & $x$ & & & & $\mathbf{x}$ & & \\
\hline \multicolumn{9}{|l|}{ ASSESSMEMNTS } \\
\hline \multicolumn{9}{|l|}{ Reviews } \\
\hline Clinical review - doctor & $x$ & & $x$ & $\mathrm{x}$ & $\mathrm{x}$ & $x$ & & \\
\hline Clinical review - nurse & & $\mathrm{x}$ & $\mathrm{x}$ & $\mathrm{x}$ & $\mathrm{x}$ & $\mathrm{x}$ & $\mathrm{x}$ & $\mathrm{x}$ \\
\hline Documented disease status & & & & $\mathrm{x}$ & & $\mathrm{x}$ & & \\
\hline \multicolumn{9}{|l|}{ Measures } \\
\hline ASSIST & $\mathrm{x}$ & & & & & & & \\
\hline ESAS & $\mathrm{x}$ & & $\mathrm{x}$ & $\mathrm{x}$ & $\mathrm{x}$ & $\mathrm{x}$ & & $\mathrm{x}$ \\
\hline AKPS & $\mathrm{x}$ & . & $\mathrm{x}$ & $\mathrm{x}$ & $x$ & $\mathrm{x}$ & & \\
\hline RUG-ADL & & $\mathrm{x}$ & $x$ & $\mathrm{x}$ & $\mathrm{x}$ & $\mathrm{x}$ & & \\
\hline CGI-I & & & $x$ & $x$ & $\mathrm{x}$ & $x$ & & \\
\hline DASS 21 & & $\mathrm{x}$ & & $\mathrm{x}$ & & $\mathrm{x}$ & & \\
\hline SLUMS & $\mathrm{x}$ & & & $\mathrm{x}$ & & & & \\
\hline EORTC QLQ-C15-PAL & & $\mathrm{x}$ & & $\mathrm{x}$ & & $\mathrm{x}$ & & \\
\hline OME & & $\mathrm{x}$ & $\mathrm{x}$ & $\mathrm{x}$ & $\mathrm{x}$ & $\mathrm{x}$ & & \\
\hline Adverse Events & & $x$ & $x$ & $\mathrm{x}$ & $\mathrm{x}$ & $x$ & $x$ & $x$ \\
\hline Concurrent medications & & $\mathrm{x}$ & $x$ & $\mathrm{x}$ & $x$ & $\mathrm{x}$ & $\mathrm{x}$ & $\mathrm{x}$ \\
\hline Vital Signs & & $x$ & $x$ & $x$ & $x$ & $x$ & & \\
\hline
\end{tabular}

The ESAS is a 9-item inventory rated on an 11-point scale anchored at 0 (no problem) to 10 (worst problem). It assesses both physical and psychological symptoms, plus general wellbeing. It has been validated in the assessment of symptoms in cancer patients [13].

Secondary outcomes include:

1. Patient-determined effective dose of THC/CBD formulation, defined as the dose that achieves symptom relief with acceptable side effects.

2. ESAS TSDS at days 7, 21 and 28.

3. Physical and emotional ESAS scores at each time point.

- Physical scores will be measured by the Australia-modified Karnofsky Performance Status (AKPS) and Resource Utilisation GroupsActivities of Daily Living Scale (RUG-ADL). The AKPS is a validated variant of the Karnofsky Performance Status. The Australian version can be applied to both in- and outpatients and is sensitive to changes in function over time [14]. The RUG-ADL is an instrument developed for the measurement of nursing dependency. The ADL scale measures patients' needs for assistance in activities of daily living (eating, bed mobility, transferring and toileting) [17].

4. Individual symptom scores (descriptive analysis only).

5. Oral morphine equivalent (OME), average use at baseline and weekly.

6. Patient Global Impression of Change (PGIC), days 7, 14, 21 and 28.

- This is a subjective measure of symptom change completed by the patients themselves [18].

7. Clinical Global Impressions (CGI) Scale, days 7, 14, 21 and 28.

- This is a subjective measure of symptom change completed by the investigating clinician [18].

8. The Depression, Anxiety, Stress Scale (DASS-21) score baseline and days 14 and 28.

- DASS-21 is a self-reported 21-item scale, 7 questions per sub-item questionnaire measuring depression, anxiety and stress. The DASS-21 will be used for assessment at baseline (day 0) and days 14 and 28 [19].

9. EORTC score QoL baseline and days 14 and 28 [20].

This is a QoL measure found valid for use in a wide variety of cancer populations. To reduce patient 
burden, we will use the 15-question subset commonly used for the palliative care population.

10. NCI common terminology for adverse events V4.03, days 2, 4, 7, 9, 11, 14, 16, 18, 21, 23, 25 and 28 [21]

- The NCI CTCAE is a severity grading system for adverse events. Adverse events relate to an unfavourable or unintended sign, symptom or disease temporarily associated with a medical treatment or a procedure which may not be related to the medical treatment or procedure. The CTCAE has a grading of 1 (mild) to 5 (death). The known common adverse events associated with cannabinoids are confusion, somnolence, paranoia, anxiety, mood changes, psychosis, hypertension, tachycardia, hyperhidrosis, nausea, vomiting and abdominal pain [21].

The study will assess adverse events (AE) and serious adverse events (SAE) using the criteria of the NCI CTCAE V4.0 [21]. The known common AEs associated with THC/CBD will be specifically addressed at each time point. Finally, a detailed concurrent medication list is to be updated and recorded at baseline (day 0) and days $7,14,21$ and 28 . This is to include over-thecounter medications, prescribed medications and complementary medication.

\section{Statistical analysis and sample size}

Allowing 20\% for attrition, and with improvement of $\geq 6$ for arm 1 compared to placebo, it is anticipated that 150 participants (75 per arm) should be randomised to achieve a sample size of 60 participants per arm, assuming 80\% power, a 1:1 randomisation scheme and a type 1 error of 5\% (two-tailed), and a standard deviation of 11.6. The sample size is based on previous work by Hui et al. [13] who determined the minimal clinically important difference in the TSDS to be 5.7 [13]. As such, we have elected to use an improvement of the TSDS of $\geq 6$ as a clinically significant change. Stata software (StataCorp. 2013. Stata Statistical Software: Release 13. College Station, TX: StataCorp) was used to estimate the sample size.

The superiority of arm 1 compared to placebo will be tested by comparing the response to each arm after 14 days, relative to baseline.

Descriptive analyses and frequency distributions will be generated from participants' demographic and clinical characteristics, with all variables explored using graphical methods and summary statistics. For the primary outcome, generalised estimating equations with the appropriate link function will be developed to assess the effect of treatment adjusted for centre. For secondary outcomes, where measurements from individual subjects are recorded several times over the course of the study, change over time in the various secondary outcome measures will be assessed using mixed models, accounting for within-subject correlation and effects due to centre. For all statistical analyses, any influence of potential covariates, for example age, sex, type of cancer and disease severity, will be explored. This study is powered to detect superiority of arm 1 over placebo.

An interim analysis will be performed after 50\% participants have completed 14 days of the trial. The analysis will be performed by an independent biostatistician blinded for the treatment allocation and reported to the investigators and the Data Safety Monitoring Board (DSMB). The purpose of the interim analysis is primarily to monitor and ensure safety of participants rather than evidence of such benefit that early stopping of the trial is justified. AEs and SAEs will be stratified by type and severity. The frequency of AEs and SAEs will be compared between treatment groups using chi-square tests and logistic regression if indicated to adjust for any baseline differences between groups. Differences in baseline characteristics are not anticipated, as the randomisation process should ensure that the patient groups are similar at baseline. However, as the numbers at interim analysis will be relatively smaller, it is possible that differences may arise by chance. All baseline characteristics will be assessed and any imbalance addressed in the analysis.

If the interim analysis shows a significant difference, the investigators and DSMB will be un-blinded to the study groups and make any stopping decision on the basis of the nature of any AEs and/or SAEs and ethical grounds, as well as consideration of any statistical differences between the groups. Grounds for stopping on evidence of clear benefit will be considered. The Peto approach will be taken with symmetric stopping boundaries at $p=0.001$.

A detailed statistical analysis plan will be prepared and ratified by the DSMB. Stata, SAS (SAS version 9.4 software (SAS Institute Inc., Cary, NC, USA)) or R ( $R$ Core Team (2017). R: A language and environment for statistical computing. R Foundation for Statistical Computing, Vienna, Austria. URL https://www. R-project.org/) will be used for analysis and all code recorded for the purposes of ensuring reproducible research.

\section{Data collection and management}

Data will be sourced from a number of modes. The study is mainly based in the outpatient clinic, so most data will be collected from the participant and recoded in the corresponding CRF or questionnaires. Some data 
will be collected from medical records, but the majority of the data will be collected from:

\begin{tabular}{|c|c|c|}
\hline Measure & Source & Collected by \\
\hline General medical information & Clinical record & Medical officer \\
\hline General demographic data & Clinical record & Study nurse \\
\hline Pathology results_-blood & Pathology report & Pathology \\
\hline Pathology results_urine & Clinical record & Study nurse \\
\hline Vital signs & CRF/clinical record & Study nurse \\
\hline $\begin{array}{l}\text { Concurrent } \\
\text { medications/OME }\end{array}$ & Clinical record & Study nurse \\
\hline $\begin{array}{l}\text { Study trial data- } \\
\text { questionnaires-ASSIST, } \\
\text { SLUMS, EORTC QLQ- } \\
\text { C15-PAL }\end{array}$ & CRF & $\begin{array}{l}\text { Patient, study nurse, } \\
\text { medical officer }\end{array}$ \\
\hline $\begin{array}{l}\text { Study trial data_ESAS, } \\
\text { AKPS, RUG-ADL, PGIC, } \\
\text { CGI-S/I, DASS-21 }\end{array}$ & CRF & $\begin{array}{l}\text { Patient, study nurse, } \\
\text { medical officer }\end{array}$ \\
\hline Side effects—safety & CRF, clinical record & $\begin{array}{l}\text { Study nurse, medical } \\
\text { officer }\end{array}$ \\
\hline
\end{tabular}

All data collected will be kept in a patient file (identified by ID number only). All data will be stored in a locked filing cabinet. At completion of the study, all CRFs will be collated and archived. Electronic files will be password protected and held within a locked office. All patient files will be reconciled and stored along with all study materials, both hard copy and electronic, consistent with the regulations of the hospital regarding the retention and disposal of patient records.

The trial will be conducted with permission and in accordance with Queensland Health regulations on the use of medical cannabis and subject to approval and monitoring by each clinical site's $\operatorname{HREC}[4,22]$. An independent DSMB to include a statistician, clinical pharmacologist, palliative care specialist and consumers will be formed and will meet regularly, with primary responsibility for monitoring adverse and serious adverse events. All AE's and SAE's will be reviewed at a minimum of 6 monthly intervals, or more frequently if needed.

\section{Post-trial care}

There is no anticipated harm or compensation for trial participation. Participants may be able to access ongoing cannabinoid products through entry into other openlabel studies but this is not guaranteed. All clinical investigators will be approved authorised cannabis prescribers and will be able to continue to prescribe, as long as the participant can fund their own supply. Participants will be informed of approved products and suppliers. The dose and formulation used post study will be at the discretion of the patient and prescriber.

\section{Discussion}

The use of cannabis for symptom control continues to be a topical issue within medicine, and this study is the first placebo-controlled, randomised trial to assess the efficacy of cannabinoids in advanced cancer patients. A major strength of this study is that it will target symptom burden as a whole, rather than just individual symptoms, in an attempt to capture the improvement in general wellbeing reported anecdotally by many who have used cannabis [23, 24]. Randomisation with placebo is essential because of the well-documented overreporting of benefit in uncontrolled trials and high placebo response rates in cancer pain trials $[25,26]$. The trial design is pragmatic, intended to allow as wide a range of participants as possible, to enable the results to be as real-world as possible. This study also allows for some data on dosing and formulation of THC/CBD specifically.

\section{Trial status}

Trial recruitment started on 09 September 2019 with protocol version number 1.3 (protocol date 30 August 2019). Recruitment is expected to continue until the end of 2021.

\section{Abbreviations}

AE: Adverse events; AKPS: Australia-modified Karnofsky Scale; ALT: Alanine aminotransferase; ASSIST: Alcohol, Smoking and Substance Involvement Screening Test; AST: Aspartate aminotransferase; BD: Twice daily; CBD: Cannabidiol; CGI: Clinical Global Impressions Scale;

CINV: Chemotherapy-induced nausea and vomiting; CPMP/ICH/135/95: Note for Guidance on Good Clinical Practice; CRF: Case report form; CRP: Creactive protein; DASS-21: The Depression, Anxiety, Stress Scale; DSMB: Data Safety Monitoring Board; EORTC QLQ-C15-PAL : European Organisation for Research and Treatment of Cancer; ESAS: Edmonton Symptom Assessment Scale; GCCS: Gold Coast Cancer Services; LGP: Little Green Pharma; GMP: Good manufacturing practice; HREC: Human Research Ethics Committee; ID: Identification; MANE: Morning; MML: Mater Misericordiae Limited; MRFF: Medical Research Future Fund; NHMRC: National Health and Medical Research Council; NOCTE: At night; OME: Oral morphine equivalent; QoL: Quality of life; QPCRG: Queensland Palliative Care Research Group; RUGADL: Resource Utilisation Groups-Activities of Daily Living Scale; SAE: Serious adverse events; SLUMS: St Louis University Mental Status exam; TDS: Three times a day; TGA: Therapeutic Goods Administration; THC: Delta-9tetrahydrocannabinol; TSDS: Total Symptom Distress Score

\section{Acknowledgements}

Not applicable

\section{Authors' contributions}

Conception/design of work-PG, AH, JM, PY, RG and JH. Drafting/revision of the manuscript-PG, AH, GG, JM, PY, RG and JH. All authors have read and approved the manuscript.

\section{Funding}

Funding has been granted through the National Health and Medical Research Council (NHMRC), acting as grant administrator for the Medical Research Future Fund (MRFF) as of January 2018.

The funder had no role in the design nor interpretation of results.

The medicinal cannabinoids to be used in this study are to be supplied by Little Green Pharma. Little Green Pharma will not be involved in the protocol development, interpretation, publication or dissemination of results. 


\section{Availability of data and materials}

The datasets used and/or analysed during the current study are available from the corresponding author on reasonable request.

\section{Ethics approval and consent to participate}

Central ethical approval has been confirmed from the Mater Misericordiae Ltd. Human Research Ethics Committee (on 14 February 2019, reference number HREC/MML/49348 (V1)), and we will not begin recruiting at other centres in the trial until local ethical approval has been obtained. This study will be conducted in compliance with all stipulations of this protocol, the condition of the ethics committee approval, the NHMRC National Statement of Ethical Conduct in Human Research (2007), the note of Guidance of Good Clinical Practice (CPMP//CH-135/95), the Australian code for the Responsible Conduct of Research (2007) and all other relevant guidance documents and legislation. The investigators/researchers will also comply with their respective professional Codes of Conduct whilst conducting this research, as well as their relevant institutional policies and procedures.

The study will be overseen by a data safety monitoring committee consisting of an independent statistician, clinicians, experts in pharmacy and drug addiction and consumer representatives.

Regular update reports will be provided to the primary funding body, the Medical Research Future Fund, and to local ethics committees at each site. Results will be submitted to a peer review journal.

All participants will give written informed consent to participate in the trial.

\section{Consent for publication}

Not applicable

\section{Competing interests}

No competing interests to declare.

\section{Author details}

${ }^{1}$ Mater Health Services, Mater Research Institute-University of Queensland, Brisbane, Australia. ${ }^{2}$ School of Pharmacy, Menzies Health Institute Queensland, Griffith University, Gold Coast, Australia. ${ }^{3}$ Mater Research Institute, University of Queensland, Brisbane, Australia. ${ }^{4}$ Greenslopes Private Hospital, Brisbane, Gold Coast Health Service, Gold Coast, Australia. ${ }^{5}$ The Australian Centre for Cannabinoid Clinical and Research Excellence (ACRE), Newcastle, Australia. ${ }^{6}$ Centre for Drug Repurposing and Medicine Research, University of Newcastle, Callaghan, Australia. ${ }^{7}$ School of Nursing, Queensland University of Technology, Brisbane, Australia. ${ }^{8}$ Centre for Palliative Care Research and Education, Queensland Health, Brisbane, Australia. ${ }^{9}$ Mater Health Services, Mater Research Institute-University of Queensland, St Vincent's Private Hospital, Brisbane, Australia.

Received: 12 October 2019 Accepted: 19 June 2020

Published online: 06 July 2020

\section{References}

1. Singer $A E$, Meeker D, Teno JM, Lynn J, Lunney JR, Lorenz KA. Symptom trends in the last year of life from 1998 to 2010: a cohort study. Ann Intern Med. 2015;162(3):175-83.

2. Yates P. Symptom management and palliative care for patients with cancer. Nurs Clin North Am. 2017;52(1):179-91.

3. Whiting PF, Wolff RF, et al. Cannabinoids for medical use: a systematic review and meta-analysis. JAMA. 2015;313(24):2456-73.

4. Qld Government. Public Health (medicinal cannabis) Act 2016. https://www. legislation.qld.gov.au/view/htmL/asmade/act-2016-053. Accessed 27 Sep 2017.

5. Elsohly MA, Slade D. Chemical constituents of marijuana: the complex mixture of natural cannabinoids. Life Sci. 2005;78(5):539-48.

6. Health Canada. Cannabis (marihuana, marijuana) and the cannabinoids (2013). http://www.hc-sc.gc.ca/dhp-mps/marihuana/med/infoprof-eng.php. Accessed 27 Sep 2017.

7. Zuardi AW. Cannabidiol: from an inactive cannabinoid to a drug with wide spectrum of action. Rev Bras Psiquiatr. 2008;30(3):271-80.

8. Zhornitsky S, Potvin S. Cannabidiol in humans-the quest for therapeutic targets. Pharmaceuticals (Basel). 2012;5(5):529-52.

9. Niesink RJ, van Laar MW. Does cannabidiol protect against adverse psychological effects of THC? Front Psychiatry. 2013;4:130.
10. National Academies of Sciences, Engineering, and Medicine. The health effects of cannabis and cannabinoids: the current state of evidence and recommendations for research. 2017.

11. Howard P, Twycross R, Shuster J, Mihalyo M, Wilcock A. Cannabinoids. J Pain Symptom Manag. 2013;46(1):142-9.

12. National Consensus Project for Quality Palliative Care. Clinical Practice Guidelines for Quality Palliative Care. 3rd ed. Pittsburgh, PA: National Consensus Project for Quality Palliative Care; 2013.

13. Hui D, Shamieh O, Paiva CE, Khamash O, Perez-Cruz PE, Kwon JH, et al. Minimal clinically important difference in the physical, emotional, and total symptom distress scores of the Edmonton symptom assessment system. J Pain Symptom Manag. 2016;51(2):262-9.

14. Abernethy AP, Shelby-James TM, Fazekas BS, Woods D, Currow DC. The Australian-modified Karnofsky Performance Status (AKPS) scale: a revised scale for contemporary palliative care clinical practice. BMC Pall Care. 2005;4:7.

15. Humeniuk R, Ali R, Babor TF, Farrell M, Formigoni ML, Jittiwutikarn J, De Lacerda RB, Ling W, Marsden J, Monteiro M, Nhiwatiwa S, Pal H, Poznyak V, Simon S. Validation of the alcohol, smoking and substance involvement screening test (ASSIST). Addiction. 2008;103:1039-47.

16. Tariq SH, Tumosa N, Chibnall JT, Perry HM III, Morley JE. The Saint Louis University Mental Status (SLUMS) Examination for detecting mild cognitive impairment and dementia is more sensitive than the Mini-Mental Status Examination (MMSE) - a pilot study. Am J Geriatr Psych. 2006;14:900-10.

17. Williams BC, Fries BE, Foley WJ, Schneider D, Gavazzi M. Activities of daily living and costs in nursing homes. Health Care Financ Rev. 1994;15(4):117-35.

18. Busner J, Targum SD. The clinical global impressions scale: applying a research tool in clinical practice. Psychiatry (Edgmont). 2007;1;4(7):28.

19. Johnson CE, Bennett KS, Newton J, McTigue J, Taylor S, Musiello T, Lau PKH. A pilot study to assess the validity of the DASS-21 subscales in an outpatient oncology population. Psycho-Oncology. 2018;27:695-9.

20. Aaronson NK, et al. The European Organization for Research and Treatment of Cancer QLQ-C30: a quality-of-life instrument for use in international clinical trials in oncology. J Natl Cancer Inst. 1993;85(5):365-76.

21. US Department of Health Human Services. Common terminology criteria for adverse events (CTCAE) version 4.0. Washington: National Cancer Institute; 2009.

22. Qld Health. Clinical guidance: for the use of medicinal cannabis products in Queensland 2016. https://www.health.qld.gov.au/_data/assets/pdf_file/ 0023/634163/med-cannabis-clinical-guide.pdf. Accessed 27 Sep 2017.

23. Luckett T, Phillips J, Lintzeris N, Allsop D, Lee J, Solowij N, et al. Clinical trials of medicinal cannabis for appetite-related symptoms from advanced cancer: a survey of preferences, attitudes and beliefs among patients willing to consider participation. Intern Med J. 2016;46(11):1269-75.

24. Swift W, Gates P, Dillon P. Survey of Australians using cannabis for medical purposes. Harm Reduction J. 2005;2:18.

25. Kunz R, Oxman AD. The unpredictability paradox: review of empirical comparisons of randomised and non-randomised clinical trials. BMJ. 1998; 317(7167):1185-90.

26. Hardy J, Quinn S, Fazekas B, Plummer J, Eckermann S, Agar M, et al. Randomized, double-blind, placebo-controlled study to assess the efficacy and toxicity of subcutaneous ketamine in the management of cancer pain. J Clin Oncol. 2012;30(29):3611-7.

\section{Publisher's Note}

Springer Nature remains neutral with regard to jurisdictional claims in published maps and institutional affiliations.

Ready to submit your research? Choose BMC and benefit from:

- fast, convenient online submission

- thorough peer review by experienced researchers in your field

- rapid publication on acceptance

- support for research data, including large and complex data types

- gold Open Access which fosters wider collaboration and increased citations

- maximum visibility for your research: over $100 \mathrm{M}$ website views per year

At BMC, research is always in progress.

Learn more biomedcentral.com/submissions 